\title{
Eine Zeitschrift schreibt Literaturgeschichte
}

\author{
Die Neue Schweizer Rundschau als Medium einer europäischen Literatur
}

\author{
Stephan Kammer
}

I.

Seit einigen Jahren schon ist die Zeitschrift als Forschungsgegenstand kein Privileg der Publizistik mehr. Lange pflegte diese mit terminologisch zwar halbwegs einleuchtenden, analytisch aber aufgrund ihrer mannigfachen Unschärfen nicht besonders erfolgversprechenden Beschreibungskategorien zu arbeiten, die Zeitschriften etwa an ihre Erscheinungsform (,Periodizität'), an die Art und Weise ihrer Verbreitung (,Publizität') sowie die ,Aktualität‘ ihrer Gegenstände banden. ${ }^{1}$ Eine literatur-, medien- und kulturgeschichtliche Zeitschriftenforschung setzte sich in den letzten Jahrzehnten von diesen Traditionen ab und begann, funktionale Kriterien für die Beschäftigung mit ihrem Gegenstand zu entwickeln und zu verwenden. Problematisierungen eines medialen Formats statt Typologie versprach diese Neubesinnung; und sie zeitigte bemerkenswerte Einsichten bereits da, wo die alten quasiontologischen Zeitschriftenmerkmale einer funktionalen und dabei historisierenden Relektüre unterzogen wurden: Publizität konnte dann als Veralltäglichung des Typographeums betrachtet werden, Aktualität eröffnete die Möglichkeit eines „Gegenwartswissen[s]“, Periodizität die einer „medienspezifisch generierte[n] Erwartbarkeit“.2 Damit wurden Ausdifferenzierung

1 Vgl. Hans Bohrmann, „Theorien der Zeitung und der Zeitschrift“, in: Joachim-Felix Leonhard et al. (Hrsg.), Handbücher zur Sprach- und Kommunikationswissenschaft, Bd. 15.1: Medienwissenschaft. Ein Handbuch zur Entwicklung der Medien und Kommunikationsformen, Berlin, New York: de Gruyter 1999, S. 143-148, hier S. 146: „Gravierend war in diesem Zusammenhang [...], daß die Merkmale als quasiüberzeitliche Konstanten aufgefaßt wurden und sich deshalb nicht eben günstig eigneten, um die Pressegeschichte von 300 Jahren angemessen auf den Begriff zu bringen. Hinzukam, daß dieser Zugriff statisch war, in sich keine Möglichkeit besaß, das Zusammenwirken, die Funktionsweise bestimmter Merkmalskonstellationen angemessen zu formulieren.“

2 Hedwig Pompe, „Botenstoffe - Zeitung, Archiv, Umlauf“, in: Hedwig Pompe und Leander Scholz (Hrsg.), Archivprozesse. Die Kommunikation der Aufbewahrung, Köln: DuMont 2002, S. 121-154, hier S. 122f. 
und Diversifizierung die interesseleitenden Fluchtpunkte einer ,Problemgeschichte ${ }^{3}{ }^{3}$ die selbst auf die unterschiedlichsten und dementsprechend disziplinübergreifenden Aspekte fokussieren konnte - sei es die Positionierung in der diskursiven Öffentlichkeit beziehungsweise im ,literarischen Feld', die für jedes Zeitschriftenunternehmen substantiell, da buchstäblich überlebenswichtig ist, sei es die Abgrenzung zu konkurrierenden Formaten des Drucks Buch und Zeitung - oder später anderer Medien, sei es die Korrelation mit Produktions- und Reproduktionstechniken (Papierherstellung, Satz, Druck). Zeitschriften, die es dementsprechend nur noch im Plural geben kann, stehen stets in Beziehung zum gesamten Problemspektrum jenes Bedingungsgefüges, von dem her sich die Produktions-, Distributions- und Rezeptionsgepflogenheiten neuzeitlicher (insbesondere, aber keineswegs nur literarischer) Öffentlichkeit verstehen lassen. Zeitschriften sind damit von Grund auf mehr als Gefäße für die beiläufigen Schreibtischgeburten moderner Autoren - Texte, die man oft als modernisierte, das heißt den Bedingungen ausdifferenzierter, ökonomisierter Schriftstellerei geschuldete Form der Gelegenheitsliteratur verstanden hat. Es gibt keine triftigen Gründe, die Rolle von Zeitschriftenpublikationen auf solchen publizistischen Notbehelf zu beschränken, auf eine Art von "steinige[m] Umweg des Autors über den Markt zum Buch als Werk $^{44}$ - zum Buch also, das dann mit einer so unreflektierten wie weitreichenden Normativität zum ,eigentlichen' Format einer Literatur im neuzeitlichen Verständnis erhoben wird.

Nicht zuletzt die (wenngleich im Einzelnen und als Begriff selbst noch zu präzisierende) „Kollektivitätvon Herausgeberschaft, Redaktion und Beiträgern“ hat der Forschungsüberblick von Frank, Podewski und Scherer als einen der aussichtsreichsten Gegenstände einer solchermaßen neu ausgerichteten, medienund kulturhistorischen Zeitschriftenforschung genannt; solche Kollektivität formiert ein systemisch-historisches „Apriori der Artikulation“, das der Autor-, Werk- und Textzentrierung herkömmlicher Literaturgeschichten ebenso unzugänglich bleibt wie dem strikt funktionalen Fokus auf das ,Sagbare' von Diskursen. ${ }^{5}$ Selbstverständlich handelt es sich dabei keineswegs um eine exklusive Bestimmung solcher Kollektivität in Sachen Zeitschrift; es wären

3 Diesen Leitbegriff entnehme ich dem auch zehn Jahre nach seinem Erscheinen noch unverzichtbaren und außerordentlich anregenden Forschungsaufriss von Gustav Frank, Madlen Podewski und Stefan Scherer, „Literatur - Zeit - Schrift. Literatur- und Kulturzeitschriften als ,kleine Archive“', in: Internationales Archiv für Sozialgeschichte der deutschen Literatur 13 (2009), S. 1-45.

4 Ebd., S. 10.

5 Ebd., S. 3 und S. 13. 
also weitere medientechnische, ökonomische, auch aufmerksamkeitsökonomische Beiträge zu derartigen Kollektiven miteinzubeziehen und mit zu bedenken. Aber als heuristische Leitplanke für die folgenden Überlegungen mag diese Bestimmung ausreichen, gerade wenn man sich mit dem Begriff der ,Autorschaft' abmüht. Denn so schon wird deutlich genug, dass Zeitschriften nur als Gegenstand einer Philologie der Kollektive beziehungsweise des Kollaborativen angemessen zu verstehen sind. Wie so viele andere Schreiboder Autorschaftskollektive sind diejenigen von Zeitschriften dabei in der Regel einerseits mehr oder weniger situativ, flüchtig, temporär - keine dauerhaften oder vielleicht genauer formuliert: strukturell zeitlosen Assoziationen also, sondern zeitweilige Konstellationen unterschiedlicher Akteure. Andererseits und im Unterschied zu personalen Kollektiven aber erzeugen und nutzen Zeitschriften durchaus Institutionalisierungseffekte, die weit über diejenigen der Autorschaftsfunktion hinausgehen und nicht mit diesen deckungsgleich sind. Zeitschriften bilden somit temporäre Institutionen der Literatur.

\section{II.}

Die Neue Schweizer Rundschau, die der Untertitel meines Beitrags als Beispiel nennt und die ich gleichsam als Testfall behandeln will, hieß nicht immer so. Sie hatte dementsprechend in den 192oer Jahren, auf die ich mich im Folgenden konzentriere, schon eine publizistische und konzeptuelle Vorgeschichte aufzuweisen. Zwar ist diese Vorgeschichte nicht ganz so lang wie diejenige der beiden deutschen Zeitschriften, zu denen sich der neue Titel wohl ins Verhältnis setzen sollte. Trotzdem mag deren Status und Relevanz dabei helfen, die im Folgenden zu umreißende Programmatik des Schweizer Parallelunternehmens zu konturieren und zu kontrastieren.

Julius Rodenbergs Deutsche Rundschau wurde 1874 ins Leben gerufen; die von Otto Julius Bierbaum gegründete, exakt 20 Jahre jüngere Neue deutsche Rundschau wurde ab 1904 und unter der Leitung Oskar Bies als Die neue Rundschau weitergeführt. Wenn das mediale Format der Kulturzeitschrift für sich genommen in den letzten Jahrzehnten des Jahrhunderts massiv an Bedeutung gewann, dann hatten gerade diese beiden Periodika sich schnell als maßgebliche kulturelle und insbesondere literarische Diskussions- und Publikationsforen des ,Kommunikationssystems Bildungsbürgertum' (Rüdiger vom Bruch) etabliert. Vor allem die Deutsche Rundschau wurde nicht nur dem Namen, sondern auch ihrem Anspruch nach „zum Modell für viele nachfolgende Kulturzeitschriften“, selbst wenn man deren (kultur)politische 
und ästhetische Konkretisierung nicht zu teilen beabsichtigte. ${ }^{6}$ Rodenbergs nicht gerade bescheidener Gründungsanspruch, seine Zeitschrift solle das „repräsentative Organ“ für alles werden, „was der deutsche Geist überhaupt ist und vermag ${ }^{\text {" }}, 7$ mochte anmaßend klingen, erfuhr aber medienstrukturell durchaus eine gewisse Realisierung. Wurde angesichts der Namenspatinnen unserer Zeitschrift, vor allem von Rodenbergs Deutscher Rundschau behauptet, „dass die Idee Kulturzeitschrift an sich ein Spezifikum des Deutschen Kaiserreichs und ihrer bildungsbürgerlichen Trägergruppen war", 8 dann dürfte das allerdings nicht nur wegen des unklaren grammatischen Bezugs des Possessivpronomens überzeichnet sein. Auf gründerzeitlichen Reichsenthusiasmus kann ein derartiges Vorhaben jedenfalls verzichten - und es ist ja nun auch nicht so, dass die publizistische Institution der Kulturzeitschrift, ja selbst des spezifischeren ,Rundschau'-Formats eine ausschließlich deutsche Erfindung gewesen wäre. ${ }^{9}$ Angesichts der eigenen Vorgeschichte der Neuen Schweizer Rundschau allerdings ist die Titeländerung dennoch signifikant.

Die Zeitschrift, die ab 1925 Neue Schweizer Rundschau heißen wird, hatte der Romanist Ernest Bovet 1907 als Wissen und Leben begründet. ${ }^{10}$ Bovet war von

6 Margot Goeller, Hüter der Kultur. Bildungsbürgerlichkeit in den Kulturzeitschriften ,Deutsche Rundschau' und ,Neue Rundschau', Frankfurt/Main u.a.: Lang 2011, S. 31. „Gerade weil für die ,Deutsche Rundschau' bürgerliche Kultur und nationale Idee nur als Synthese denkbar waren, arrangierte sie sich in harmonisierender Obrigkeitstreue mit dem wilhelminischen Machtstaat und dessen öffentlicher Nationalkultur, entfernte sich allerdings zusehends - wie die Nationalliberalen insgesamt - von den emanzipatorischen Idealen eines liberalen Rechts- und Kulturstaats“ (ebd., S. 180).

7 Zit. n.Julius Rodenberg, Die Begründung der „Deutschen Rundschau“. Ein Rückblick, Berlin: Paetel 1899, S. 29.

8 Goeller, Hüter der Kultur (Anm. 6), S. 3 .

9 Rodenberg orientierte sich formatbezogen an den britischen und französischen Reviews bzw. Revues des 19. Jahrhunderts: „Die Elemente der ,Revue bestimmten [...] den redaktionellen Aufbau seines Zeitschriftenmodells: enzyklopädische Orientierung, Besprechung nationaler und internationaler Ereignisse und Entwicklungen im kulturellen und politischen Bereich und an zeitgenössischen Bildungsidealen orientierte Vermittlung zwischen wissenschaftlicher Erkenntnis und kultureller Praxis auf dem Gebiet der schönen Künste, wobei die Textform dem literarischen Ausdruck in Originalbeiträgen und Kritik entgegenkam“ (Karl Ulrich Syndram, „Rundschau-Zeitschriften. Anmerkungen zur ideengeschichtlichen Rolle eines Zeitschriftentyps“, in: Ekkehard Mai, Stephan Waetzoldt und Gerd Wolandt (Hrsg.), Ideengeschichte und Kunstwissenschaft. Philosophie und bildende Kunst im Kaiserreich, Berlin: Gebr. Mann 1983, S. 349-370, hier S. 357 .

10 Zwei Aufsätze haben sich bisher mit der Geschichte der Zeitschrift beschäftigt. Beide widmen sich allerdings der Periode vor dem Namenswechsel: Hans Ulrich Jost, ,"Wissen und Leben ( oct. 1907-déc. 1925)“, in: Les Annuelles 4 (1993): Histoires des revues, hrsg. von 
1902 bis 1922 Ordinarius für italienische und französische Literaturgeschichte an der Universität Zürich. ${ }^{11}$ Während dem im selben Zug und unter demselben Namen ins Leben gerufenen Verein wenig Erfolg beschieden war, etablierte sich die Zeitschrift schnell als eine der gewichtigeren Kulturzeitschriften des Landes. Unter Bovets finanzieller und programmatischer Regie sollte ein lebensphilosophisch grundierter, recht buntscheckiger helvetischer Kulturidealismus propagiert werden - gewissermaßen in medialer Verlängerung seiner akademischen Lehrtätigkeit über die Universitätsmauern hinaus ins ,Leben“ wollte Bovet „die Entwicklung kräftiger, zielbewusster Individualitäten in idealistischer Richtung [...] fördern" und nahm zu diesem Zweck in seinen einleitenden Bemerkungen auch auf das Medienformat der Kulturzeitschrift und deren privilegierten Gegenstand Bezug: ${ }^{12}$

Das wird uns nicht sofort gelingen; zwischen den Mitarbeitern und den Lesern unserer Zeitschrift wird die Fühlung nur allmählich entstehen; wir wagen den Versuch in der bestimmten Hoffnung, dass der Weg uns Alle zu neuen Gesichtspunkten führen wird. Eins möchten wir schon heute betonen: die hohe Bedeutung der Kunst. Das wachsende Interesse, das man heute der Kunst entgegenbringt, ist ein weiterer Beweis für das bestehende Verlangen nach innerer Befreiung. Die tägliche Arbeit, wie redlich sie auch sei, erscheint uns oft als ein blosses Stückwerk; auf den Flügeln der Kunst erhebt sich das Gemüt über jene Enttäuschungen bis in das Reich, wo die edelsten Träume der Menschheit seit Jahrhunderten in schönen Formen ihren Ausdruck gefunden haben. Das Schöne ist ein unentbehrliches Element des Lebens; es soll nicht nur in der Musik, in der Malerei gepflegt werden; sein Licht soll alles durchdringen, auch unsere Gedanken. ${ }^{13}$

Bei allem Pathos, das die eröffnende Zielvorgabe der Zeitschrift kennzeichnet, umreißt diese doch die Konturen der anschließenden publizistischen

Alain Clavien, Diana Le Dinh und François Vallotton, S. 103-110; Peter Utz, „Anspruchsvolle Anstrengungen helvetischer ,Kulturträger. Die Anfänge der schweizerischen Kulturzeitschrift Wissen und Leben“, in: Ulrich Mölk in Zusammenarbeit mit Susanne Friede (Hrsg.), Europäische Kulturzeitschriften um 1900 als Medien transnationaler und transdisziplinärer Wahrnehmung. Bericht über das Zweite Kolloquium der Kommission ,Europäische Jahrhundertwende - Literatur, Künste, Wissenschaften um 1900 in grenzüberschreitender Perspektive, Göttingen:Vandenhoeck \& Ruprecht 2006, S. 93-112. Auf https:// www.e-periodica.ch sind mittlerweile alle Jahrgänge der Zeitschrift online zugänglich. Ich zitiere im Folgenden unter den Siglen $W L$ und NSR mit Angabe von Bandnummer, Datum und Seitenzahl.

11 Vgl. die etwas hagiographisch geratene Biographie von Georges Büttiker, Ernest Bovet. 1870-1941, Basel, Stuttgart: Helbing \& Lichtenhahn 1971.

12 Ernest Bovet, „Unser Ziel“, WL 1 (Oktober 1907), S. 1.

13 Ebd., S. 3 f. 
Aktivität recht gut - anders gesagt: bildet die Zielvorgabe trotz des Pathos einen Rahmen, in dem vielstimmige (und mehrsprachige) Aktivitäten Platz finden konnten. So „kannte der Themenkreis der Zeitschrift keine Einschränkungen; jeder durfte sich am Gespräch beteiligen, seine Meinung kund tun, auch wenn sie den Redaktoren nicht genehm, das gewählte Thema unorthodox war. ${ }^{{ }^{114}}$ Das gelang, obwohl man ökonomisch stets unter vergleichsweise prekären Bedingungen arbeitete und ohne das angeheiratete und bereitwillig ins Unternehmen eingebrachte Vermögen des Gründers (ab 1914 aus Spargründen auch Redakteurs) Bovet kaum dauerhaft funktionsfähig gewesen sein dürfte - trotz der vergleichsweise stabilen 4.0oo Abonnenten der Zeitschrift und mit einem Abonnementspreis, der laut Hans Ulrich Jost nach dem Ersten Weltkrieg dem doppelten Tageslohn eines Arbeiters entsprach, ${ }^{15}$ hatte die Zeitschrift in den ersten sieben Jahren ihres Bestehens ein jährliches Defizit von 25.00o bis 30.000 Franken zu verbuchen. ${ }^{16}$ Bovet selbst sollte bis 1918 ungefähr 200.000 Franken in das Unternehmen investieren. ${ }^{17}$

Ende 1922 übernahm der frisch promovierte Literaturhistoriker Max Rychner die Redaktion der Zeitschrift von Bovet, als dieser neben Wissen und Leben auch seine Zürcher Professur aufgab, um Generalsekretär der Schweizerischen Vereinigung für den Völkerbund zu werden. Wenn man Büttikers Biographie folgt - sie belässt es bedauerlicherweise bei ganz unspezifischen Andeutungen -, dann gelang diese Übergabe nicht ganz reibungslos. ${ }^{18}$ Er denke „in keiner Weise daran [...], von Wissen und Leben ganz Abschied zu nehmen", hatte Bovet zum Abschluss des 15. Jahrgangs und anlässlich der Redaktionsübergabe jedenfalls festgehalten und eine Balance von „Kontinuität" und "Auffrischung" in Aussicht gestellt, ${ }^{19}$ "der Schriftsteller Bovet bleibt auf seinem Posten“, versicherte eine redaktionelle Notiz Rychners noch zum Anfang des 17. Zeitschriftenjahrgangs. ${ }^{20}$ Tatsächlich trug er bis Ende des darauffolgenden Jahres auch regelmäßig zur Zeitschrift bei; nach dem

14 Büttiker, Ernest Bovet (Anm. 11), S. 29.

15 Jost, „Wissen und Leben“" (Anm. 10), S. 103.

16 So die Angaben bei der Ankündigung des Redaktionswechsels: Ernest Bovet, „Von der Zukunft unserer Zeitschrift", $W L 15$ (April 1922), S. 498.

17 Büttiker, Ernest Bovet (Anm. 11), S. 31.

18 Vgl. ebd.: „Bald musste Bovet erkennen, dass die Zeitschrift unter der neuen Führung die ursprüngliche Bahn immer mehr verliess und die treuesten Mitarbeiter [...] langsam ausgebootet wurden. Die sich abzeichnende Entwicklung betrübte ihn; da er aber die nachstossenden Kräfte nicht zurückbinden wollte, zog er die Konsequenzen und reichte seine Demission ein. Er hatte gespürt, dass auch er der neuen Redaktion lästig war, und als Geduldeter wollte er nicht mehr mitarbeiten."

19 Ernest Bovet, „Zum Schlusse des 15. Jahrgangs“, WL 15 (September 1922), S. 95 o.

20 Max Rychner, „Zum neuen Jahrgang“, WL 17 (Oktober 1923), S. 3. 
September 1925 - im letzten Jahrgang, der Wissen und Leben als Haupttitel führt - allerdings findet man keinen seiner Artikel mehr. Die lakonische, von der "Redaktionskommission“ institutionell unterzeichnete Begründung des angekündigten Titelwechsels liest sich in dieser Hinsicht tatsächlich zwiespältig. Die Pointe, der alte Titel habe „Nichtkenner oft dazu verführt, in unserer Zeitschrift eine naturwissenschaftliche Publikation zu vermuten“, dürfte bestenfalls ironisch zu verstehen sein. Die Anmerkung aber, „der Titel Neue Schweizer Rundschau“ gebe "für alle, die unser Organ nicht kennen, besonders für Ausländer mehr Aufschluss über die innere Gestalt" der Zeitschrift als der bisherige Name, versteht sich in dem eben skizzierten Kontext der RundschauZeitschriften ohne Weiteres. ${ }^{21}$ Die für die ,Rundschaupublizistik' einschlägige „inhaltliche Mischung aus literarischen Originalbeiträgen, wohlwollend vorstellender Kritik eigener Autoren und längeren Aufsätzen zu Sachfragen“22 jedenfalls hatte bereits Rychners zitierte Annonce vom Herbst 1923 in Aussicht gestellt, die der Literatur den ihr gebührenden „Platz an der Sonne“ in der reorganisierten Zeitschrift versprach. ${ }^{23}$

III.

Systematisierbare Konturen erhielten solche mehr oder weniger subtilen Veränderungen allerdings nur, wenn man die redaktionelle Akzentverschiebung gleichzeitig summarisch erfasste und im Einzelnen nachverfolgte. $\mathrm{Zu}$ diesem Zweck wäre die von Frank, Podewski und Scherer angemahnte Fokussierung auf das „komplexe Funktions- und Formenbündel“ der Zeitschrift zu konkretisieren. ${ }^{24}$ Welche mobilen Kollaborationen von Verfassern, redaktionellen Vorgaben, Textsorten, Themen und Korrespondenzen waren es, mittels derer sich diese Verschiebungen artikulierten? Wie stabil waren diese publizistischen Institutionalisierungen? Welche Improvisationsspielräume erlaubten sie - beziehungsweise: benötigten sie, um die nötige Balance von Aktualität und Erwartbarkeit zu gewährleisten? Dass ich auf diese Fragen im Folgenden nur einige Schlaglichter werfen kann, liegt nicht nur am begrenzten

\footnotetext{
21 „An unsere Abonnenten“, WL 18 (Dezember 1925), S. 1173.

22 Syndram, „Rundschau-Zeitschriften“ (Anm. 9), S. 353.

23 Rychner, „Zum neuen Jahrgang“ (Anm. 20), S. 3. Mit der Heraushebung der Literatur überführt, könnte man vielleicht behaupten, die neue Redaktion die Zeitschrift vollends ins ,Rundschau'-Dispositiv, in dem literarische Texte die bevorzugte künstlerische Form darstellen. Bovet hat, obwohl selber Literaturwissenschaftler, der bildenden Kunst bzw. der Musik tendenziell den Vorzug gegeben.

Frank/Podewski/Scherer, „Literatur - Zeit - Schrift“ (Anm. 3), S. 32.
} 
Umfang; auch nicht daran, dass wenigstens ein Teil der Antworten darauf auf eine diskursiv nur schwer vermittelbare Quantifizierung von Beiträgen und Beiträgern zurückgreifen müsste und ich an dieser Stelle ohnehin nicht mit einem illustren und nicht ganz so illustren name dropping von Richard Alewyn bis Albin Zollinger langweilen möchte: Das Netzwerk einer Zeitschrift beschränkt sich ja nicht auf die beteiligten personalen Akteure. Vielmehr stellt eine Gesamtanalyse dieser Zeitschriftenkollaboration und ihrer Artikulationen zur Zeit schlicht ein noch ungedecktes Versprechen dar - kurzum: sie ist Projektskizze und Aufgabenstellung, die zu ihrer Realisierung noch ein paar Hürden nehmen muss, institutionelle und konkret arbeitstechnische gleichermaßen. Ausgangsvoraussetzung und Ziel dieses Unternehmens zugleich muss dabei aber nichts anderes als das eben noch einmal Angesprochene sein: einen bedeutsamen Ausschnitt der Literaturgeschichte der europäischen Moderne als Produkt eines mobilen Kollektivs von Verfassern, Redakteuren, Textsorten, Themen und Korrespondenzen zu verstehen, anstatt sie an der Enfilade von Autoren und Werken, Wirkung und Einflüssen zu buchstabieren. Die Zeitschrift Wissen und Leben (alsbald ergänzt durch den Untertitel Neue Schweizer Rundschau, schließlich von diesem abgelöst) hat in den Jahren zwischen 1922/23 und 1931 als teils mobiles, teils stabiles Kollektiv einem wesentlichen Teil der literarischen Moderne publizistische Konkretion verschafft. Die Zeitschrift bildet eine medial gebundene Konstellation von Artikulationen, die weder bloß Aggregat einzelner Beiträge ist noch den emphatischen Schließungsansprüchen eines ästhetischen Werkbegriffs zu genügen braucht, sondern als repräsentativer Knoten in einem Beziehungsnetz namens Europäische Moderne betrachtet werden muss.

Ein erstes, methodologisches Schlaglicht versteht sich möglicherweise nach dem eben Angeführten fast von selbst. Ich habe unlängst den Begriff einer ,Netzwerkphilologie‘ verwendet, um mich, zugegeben: ein wenig polemisch, über die traditionsreiche und anhaltende freiwillige Selbstbeschränkung philologischer Theorie und Praxis auf Einzeltextphilologie zu wundern. ${ }^{25}$ Damit meine ich selbstverständlich keineswegs, man sollte um eines sogenannten medientechnischen Fortschritts willen oder gar aus modischen Erwägungen Abgesänge aufs Gedruckte an- und in die Flexibilisierungsrhetoriken anlässlich digitaler Textrepräsentationsmöglichkeiten einstimmen. Das wäre allein schon deshalb mehr als fahrlässig, weil digitale philologische Praxis strukturell, das heißt technisch bedingt notwendigerweise ungleich rigider auf das Prinzip

25 Vgl. Stephan Kammer, „Netzwerke edieren, oder:Was wäre der,Text'von Korrespondenzen? Einige Anmerkungen aus Anlass zweier Briefeditionen zu E.R. Curtius“, in: editio 31 (2017), S. $233-246$. 
des einzelnen Texts verpflichtet sein muss (und sei es in der unanschaulichen Form von Datensätzen), als sich das Buchnostalgiker träumen lassen. Es mag kontraintuitiv klingen, aber die performative Kontextrealisierung namens ,Hypertext' gründet auf einer weitaus strengeren Individualisierungslogik, als es das herkömmliche relationale Doppel von Text und Apparat je herzustellen vermocht hätte. Die eindeutige Adressierbarkeit einzelner Dateien ist Voraussetzung aller hypertextuellen Präsenz- und Grenzüberschreitungseffekte. Wenn ich also den Terminus einer ,Netzwerkphilologie' aufwerfe, dann geht es mir nicht in erster Linie ums Digitale oder um mediale Darstellungsgepflogenheiten. Ich will vielmehr das philologische Privileg des einzelnen Textes zur Verhandlung stellen und somit sozusagen auch am anderen Ende der Kette wiederholen, was das vorliegende Publikationsunternehmen auf Verfasserseite unternimmt. Mit am dringlichsten stellt sich das Problem des einzelnen Texts für die Provokation einer Netzwerkphilologie angesichts von Sachverhalten, denen philologisch meist ein im Wortsinn parergonaler Status zukommt. Das gilt einerseits für den Brief beziehungsweise genauer: für Briefkorrespondenzen, die von der Dominanz einer einzeltextbezogenen Theoriebildung und Praxis besonders tangiert werden. Es gilt aber selbstverständlich nicht minder für die mobilen Kollektive von Verfassern, Redakteuren, Textsorten, Themen und Korrespondenzen, die man Zeitschriften nennt. Es gilt, mit anderen Worten, wenn man Zeitschriften nicht als Kanäle für Vorabveröffentlichungen und Broterwerbskompromisse versteht, die vor beziehungsweise neben dem eigentlichen literarischen Werk anzusiedeln sind, sondern als Gegenstände eigenen - auch philologisch eigenen - Rechts.

Mit einem zweiten, bereits halb thematischen Schlaglicht möchte ich die Frage aufwerfen, ob möglicherweise der von der Neuen Schweizer Rundschau vertretene Anspruch einer europäischen literarischen Moderne nicht wenigstens eine konzeptuelle Wahlverwandtschaft zu solcherlei vielfach prekärem Zeitschriftenwesen unterhält - stellt ihm sich doch die Frage nach dem Einzelnen und dem Ganzen in Bezug auf ,Europa' nicht minder dringlich als für deren medialen Realisierungsort ,Zeitschrift'. Denn dieses ,Europa‘ der Zwischenkriegszeit ist Chronotopos und imaginäre Setzung weit mehr als Gegebenheit; es ist nur in den durchaus diversen, ja heterogenen Kontexten des jeweiligen Gebrauchs zu verstehen; es ist von zahlreichen und unterschiedlichen Beziehungen der Exklusion und Inklusion bestimmt - gleichsam das strukturelle Äquivalent zu dem ,Orient', den Edward Saids Analysen aufgeschlüsselt haben ${ }^{26}$ Auch das müsste man nun ausführlicher herausarbeiten; ${ }^{27}$

26 Vgl. Edward Said, Orientalism, New York: Pantheon 1978.

27 Erste Anmerkungen vor allem zur Vorgeschichte dieser Europa-Imaginationen in Stephan Kammer, „Hedging the Particular, Hedging the Universal: Structural ,Europes‘ in Literary 
Hugo von Hofmannsthals schon während des Krieges skizzierte ,EuropaImaginationen dürften von den bekannteren entsprechenden Artikulationen der Zeit derjenigen jedenfalls am nächsten kommen, die sich in der Neuen Schweizer Rundschau abzeichnet. ${ }^{28}$

Rychner, der 1922 mit einer Arbeit zu Georg Gottfried Gervinus promoviert wurde, waren die Argumentationslinien einer literarisch-kulturellen Vorläuferschaft und der dadurch präfigurierenden Integration einer noch nicht institutionell existierenden politischen Bezugsgröße natürlich bestens vertraut. Gervinus' Geschichte der poetischen National-Litteratur der Deutschen (1835-42) war eine der wichtigsten Stimmen, die die titelgebende Nationalliteratur als Antizipation eines noch ausstehenden, allerdings im Unterschied zum späteren Kaiserreich „liberal verfaßten Nationalstaats" propagiert hatte. ${ }^{29}$ Sein Beitrag zur "Geschichte eines politisierten Kulturbegriffs“ dürfte gewiss nicht gering zu veranschlagen sein: „Gervinus vertritt eine Literaturgeschichtsschreibung, die nicht nur den Zusammenhang zwischen Poesie und bedingender Wirklichkeit erläutert, sondern zugleich die Literatur auf ihren Wert für die Gegenwart befragt. Er will daher nicht so sehr für seine Kollegen als vielmehr für seine Zeitgenossen schreiben: nicht für die Fachwissenschaft, sondern ,für die Nation “',30 wie es in der Einleitung zu seiner Geschichte heißt.

Es spricht einiges dafür, dass in den besagten Jahren nach dem Ersten Weltkrieg die Reaktualisierung dieses Antizipationsverhältnisses im Namen Europas das zentrale Anliegen des mobilen Kollektivs der Neuen Schweizer Rundschau ist. Fände man Lust an der pointenhaften Zuspitzung, könnte man die mit der Redaktionsübergabe verbundene Neuausrichtung der Zeitschrift als funktionale Differenzierung verstehen: Während Bovet die Zeitschrift verlässt, um beim Völkerbund Europa politisch voranzutreiben, setzt Rychners

Modernism (1890-1917)“, in: Chunjie Zhang (Hrsg.), Composing Modernist Connections in China and Europe, New York, London: Routledge 2019, S. 97-119.

28 Hugo von Hofmannsthal, „Die Idee Europa. Notizen zu einer Rede“, in: ders., Gesammelte Werke in zehn Einzelbänden, Reden und Aufsätze II: 1914-1924, hrsg. von Bernd Schoeller, Frankfurt/Main: Fischer 1979, S. 43-54, sowie ders., „Über die europäische Idee“, in: ders., Sämtliche Werke. Kritische Ausgabe, Reden und Aufsätze III, hrsg. von Klaus E. Bohnenkamp, Katja Kaluga und Klaus-Dieter Krabiel, Frankfurt/Main: Fischer 2011, S. 324-335 und S. 1268-1300.

29 Wolfgang J. Mommsen, Das Ringen um den nationalen Staat. Die Gründung und der innere Ausbau des Deutschen Reiches unter Otto von Bismarck 1850 bis 189o, Berlin: Propyläen 1993 (= Propyläen Geschichte Deutschlands, Bd. 7.1), S. 713.

30 Klaus L. Berghahn, „Von Weimar nach Versailles. Zur Entstehung der Klassik-Legende im 19. Jahrhundert", in: Reinhold Grimm und Jost Hermand (Hrsg.), Die Klassik-Legende. Second Wisconsin Workshop, Frankfurt/Main: Athenäum 1971, S. 50-78, hier S. $5^{2}$ und S. 62. 
Redaktion darauf, in der Zeitschrift selber Europa als literarisches Projekt zu befördern. Ex negativo deuten bereits Bovets redaktionelle Abschiedsworte diese Verschiebung an: „Der Krieg brachte es mit sich, dass gewisse Fragen vertieft wurden, dass aber auch der Horizont sich verengerte; Kunst und Literatur waren leider nur noch Nebensache.“" ${ }^{\text {31 }}$ Unter Rychners redaktioneller Verantwortung kommen Literatur und Kunst tatsächlich massiv zurück, ohne dass allerdings die politischen und kulturpolitischen Einlassungen der Zeitschrift deswegen verschwunden wären. Vielleicht könnte man deswegen diese Pointe ebenso gut umkehren und behaupten: Eine Programmatik des europäischen ,Völkerbunds‘ wandert in den Literatur- und Kunstdiskurs der Zeitschrift ein. Es sei "gleichgültig, aus welchem Lande das Wissen [...] in die Welt gelangt, da es ja keinen Zoll bezahlt", ${ }^{32}$ behauptet zum Beispiel Carl Helbling im Juni 1925 in einem Überblicksartikel zu aktueller Forschungsliteratur über Oscar Wilde; und nur einen Monat später unterstreicht Rudolf Utzinger ähnlich beiläufig eine solche postnationale Epistemologie, „weil der kulturführende Mensch aller Nationen exterritorial ist".33

,Europa' erscheint in dieser Modellierung als Übertragungsfigur, als Figur des Dritten, die an die Stelle von zwei in der Regel gleichermaßen verworfenen Konzepten respektive Konzeptgruppen gerückt beziehungsweise zwischen diese gestellt wird und gegen deren Dichotomien ausgespielt werden kann. Zwischen den Nationalstaat und dessen ,ganz Anderes' zum Beispiel, heiße dies konkret „Amerikanismus“ und „Bolschewismus“ wie bei Ernst Robert Curtius, soll dann eine nun schon weitaus weniger konkrete "geheime geistige Aristokratie Europas" treten. ${ }^{34}$ Aber auch einem vageren, eher abstrakten kosmopolitischen ,Anderen' erteilt man Absagen im Namen Europas. Zu einer Publikation des vormaligen Jugendbewegungs-Pädagogen Gustav Wyneken, die nach den Möglichkeiten eines ,Vaterlands Europa' fragt und dessen Realisierbarkeit noch durch so einigen essentialistischen Ideologie-Ballast beeinträchtigt sieht, merkt Rychner an:

Sicherlich. Und neben den nationalen, religiösen, rassischen usw. Ideologien, die einem historischen Einzelphänomen allen Sinn zuweisen, sind es wohl nicht zuletzt weit kraftlosere Ideologien, die einen abstrahierten Internationalismus aufs Papier projizieren, wo er denn auch still verharren bleibt. ${ }^{35}$

31 Bovet, „Zum Schlusse des 15. Jahrgangs“ (Anm. 19), S. 949.

32 Carl Helbling, „Literatur über Oscar Wilde“, WL 18 (Juni 1925), S. 685.

33 Rudolf Utzinger, „Völkerkundliche Einblicke“, $W L 18$ (Juli 1925), S. 778.

34 Ernst Robert Curtius, „Restauration der Vernunft“, NSR 20 (September 1927), S. 862 und S. 859 .

35 Max Rychner, „Literarische Hinweise“, NSR 20 (Dezember 1927), S. 1140. Rychners Kurzrezensionen in dieser Nummer nehmen auch auf den wichtigen Münchner Vortrag von 
Es fehlen zwar der konzeptuellen Annäherung an dieses Dritte, das ,Europa heißen soll, belastbare Positivkriterien, und wenn Rychner in dem gerade zitierten Beitrag „Erkenntnis“, ${ }^{36}$ Curtius' Essay ,Vernunft als Heilmittel gegen die verworfenen Ideologien ins Feld führt, bleibt dies vergleichbar unscharf wie in Hofmannsthals Europa-Schriften zehn Jahre zuvor der Rekurs auf den ,Geist'. Als Ausnahme von solcher Unterbestimmtheit aber gerade soll das literarische ,Europa' gelten dürfen, dem das mobile Kollektiv der Neuen Schweizer Rundschau Platz und Aufmerksamkeit verschafft. Angesichts, großer Literatur' werden dann gelegentlich gerade für Rychner sogar die Grenzen zwischen ,Europa' und ,Welt' doch wieder durchlässig, wie die einleitenden Bemerkungen zu seinem Dostojewski-Essay von 1926 zeigen:

Wo es heute große Dichtung gibt, ist sie weltgültig und weltheimisch, und nicht erst seit heute. Shakespeare ist noch spät auf dem Kontinent durchgedrungen, aber mit Voltaire und Rousseau wird das Tempo des geistigen Umsatzes in Europa rasch. Man wird für größere Entfernungen feinhörig. Nietzsches Ruhm wächst nicht aus einem deutschen zu einem europäischen empor, er bildet sich von Anbeginn als etwas vorbestimmt und strukturhaft Europäisches. Über den Ruhm hinaus verhält es sich mit der Wirkung so. In der Gegenwart leben wir im steten Bewusstsein, es gebe ein paar europäischer Statthalter des Geistes, die unser Zeitalter ebensosehr wie ihre Nation vor der Nachwelt vertreten. Es bedurfte der Veröffentlichung eines schmalen Gedichtbandes, und die Welt wusste, auch wo sie nicht begriffen haben konnte, dass Paul Valéry zu den Unverlierbaren unseres Zeitalters gehört. Solche plötzlich sich bekundende Weltgeltung und Wirkung über den eigenen Sprachkreis hinaus haben zur Folge, dass im „nationalen“ Geistesleben Mächte Bedeutung und Einfluß erlangen, die von fernher strömen. ${ }^{37}$

Nicht zuletzt der Herstellung oder Ermöglichung solcher ,Plötzlichkeit‘ haben die Aktivitäten des mobilen Kollektivs Neue Schweizer Rundschau zu dienen. Der Name Valérys in diesem Zusammenhang fällt alles andere als zufällig: Hat doch das Januar-Heft dieses ersten Jahrgangs unter dem neuen Zeitschriftentitel mit einer Übersetzung von Valérys Essay Über die Krise der Intelligenz aufgewartet, Rychner selbst im März-Heft seine Übersetzung der Soirée avec monsieur Teste veröffentlicht und auf die anstehende Publikation

Hugo von Hofmannsthal Bezug, der zunächst in der Neuen Rundschau und dann als Sonderdruck unter dem Titel Das Schrifttum als geistiger Raum der Nation (1927) publiziert worden ist. Dass eine Zwischenüberschrift den Titel zu Das Schriftum als geistiger Raum (ebd., S. 1135) redigiert, ist gewiss kein Zufall.

36 Ebd., S. 1140.

37 Max Rychner, „Dostojewski und der Westen“, NSR 19 (Mai 1926), S. 508. 
der gesammelten Teste-Texte hingewiesen. ${ }^{38}$ Diese Valéry-Korrespondenzen bieten übrigens, dies in Klammern, den bekanntesten Beleg dafür, dass ein solches mobiles Kollektiv seine Programmatik keineswegs auf die medialen Grenzen und „die positive Gegebenheit" ${ }^{\text {“9 }}$ einer konkreten Zeitschrift beschränken muss - gerade in ihrer strukturellen Unabschließbarkeit liegt ja ein entscheidendes Kriterium der Netzwerkartigkeit, die ein solches Kollaborationsunternehmen ausmacht und in der das Medium Zeitschrift ein (wenngleich wichtiger) Knotenpunkt unter anderen ist.

\section{IV.}

Ich will abschließend mit einem dritten Schlaglicht auf das mobile Kollektiv Neue Schweizer Rundschau demonstrieren, wie wenigstens bisweilen Akteure dieses Kollektivs mit dessen Bedingungsgefüge zu spielen anfangen können. Am 18. März 1926 wendet sich Robert Walser an Rychner, mit dem er bereits seit 1923 über Publikationsbelange korrespondiert. Der Schriftsteller bedankt sich für die „Übersendung des Honorares und Heftes Ihrer Revüe“ (B 685, S. 198), ${ }^{40}$ die er sich mit dem Prosastück Die Ruine ${ }^{41}$ verdient hat - es handelt sich um das März-Heft des 19. Zeitschriftenjahrgangs, von einiger literaturhistorischer Bedeutung nicht zuletzt auch durch den erwähnten Vorabdruck aus Valérys Teste. Bekannt geworden ist Walsers Brief zunächst wegen des gleich zur Eröffnung geäußerten ironischen Verdachts, der Satz der Ruine - eines für Walsers Berner Prosa übrigens ungewohnt umfangreichen Prosastücks - sei auf honorardrückerische Intentionen des Redakteurs Rychner zurückzuführen. ${ }^{42}$

38 Paul Valéry, „Über die Krise der Intelligenz“, NSR 19 (Januar 1926), S. 7-23; ders., „Der Abend mit Herrn Teste“, NSR 19 (März 1926), S. 221-232. Die angekündigte Buchausgabe von Rychners Übersetzung erschien im folgenden Jahr: Paul Valéry, Herr Teste, Leipzig: Insel 1927 .

39 Frank/Podewski/Scherer, „Literatur - Zeit - Schrift“ (Anm. 3), S. 41.

40 Robert Walser an die Redaktion der Neuen Schweizer Rundschau/Max Rychner, 18.03.1926, in: Robert Walser, Briefe 1921-1956, hrsg. von Peter Stocker und Bernhard Echte unter Mitarbeit von Peter Utz und Thomas Binder, Berlin: Suhrkamp 2018 (= Werke. Berner Ausgabe, Bd. 2), Nr. 685, S. 198-201. Zitate aus dieser Ausgabe werden mit der Sigle B und Briefnummer sowie Seitenzahlen im laufenden Text nachgewiesen.

41 Vgl. zu diesem poetologisch wichtigen Prosastück Stephan Kammer, Figurationen und Gesten des Schreibens. Zur Ästhetik der Produktion in Robert Walsers Prosa der Berner Zeit, Tübingen: Niemeyer 2003, S. 61-73; Jochen Greven, „Poetik der Abschweifungen. Zu Robert Walsers Prosastück ,Die Ruine““, in: Wolfram Groddeck et al. (Hrsg.), Robert Walsers ,Ferne Nähe؛ Neue Beiträge zur Forschung, München: Fink 2007, S. 177-186.

$42 \ldots[\ldots]$ würde ich mir vielleicht leise wie ein Mäuslein erlaubt haben zu glauben, Sie hätten diese edle und seltsame ,Ruine` sehr eng, schlank, dünnineinandergepreßt setzen und 
Tatsächlich hat man für das Prosastück die kleinere Type und den engeren Satz verwendet, der üblicherweise den Rubriken am Heftende vorbehalten bleibt. Doch dann beginnt der Briefschreiber mit einer Revue der „Revüe“, Lob und Tadel für das aktuelle Heft zu verteilen:

Gestatten Sie mir, Ihnen auf Salonmanier anzuvertrauen, ich hätte über die Weltberühmtheit dieses Monsieur Valéry ein bischen gelächelt. Ich und einige Andere warten nun bereits auf Ablösung, d.h. auf die Absetzung des Sternes, und auf die Einsetzung oder Tronbesteigung eines frischen Weltkurfürsten. Proust und Morand sind also schon so gut wie zum Glück beseitigt. Hermannchen mit dem Beiwort Hiltbrunner besitzt zu wenig Schmalz, Schmelz, Glanz, Saft und klangliche Kraft. Sagen Sie ihm das natürlich lieber nicht, da es ihn betrüben könnte. St. Evremond ist ein sehr hübscher, interessanter Essay, den nun eine Glätterin liest, die eine meiner Freundinnen ist, und der ich Ihr Heft einsandte. Sie hat verhältnismäßig viel mit der Länge von Jahren, die sie in Walser's Gesellschaft zubrachte, sich erworbene Bildung. (B 685, S. 198f.)

Mit der „Weltberühmtheit“ nimmt Walser einen Begriff aus Rychners Übersetzernachwort auf; ${ }^{33}$ Proust (der im März-Heft nicht genannt ist) wird, als ob sich der ironische Witz dieser Korrespondenz tatsächlich fortsetzen sollte, in der ungefähr zwei Wochen nach dem Brief veröffentlichten April-Nummer der Neuen Schweizer Rundschau eine zentrale Rolle spielen: Man findet dort einen Beitrag von Ernst Howald zur Psychologie Marcel Prousts - und eine Ode an denselbigen aus der Feder des von Walser im selben Satz erledigten Paul Morand. ${ }^{44}$ Das Lob für Curtius' Saint-Evremond-Essay, der mit einer Würdigung der Liebhaberei und des Dilettantismus beginnt, spiegelt sich in der Anmerkung zur idiosynkratischen Bildungsgeschichte von Walsers Freundin und Korrespondenzpartnerin Frieda Mermet, der genannten „Glätterin“, der er das Heft weitergeschickt haben will. ${ }^{45}$

drucken lassen, damit so wenig Seitenzahl und so wenig Lohn wie möglich hervorblickte und -schaute" (B 685, S. 198). Der Witz dieser Einlassung beginnt allerdings bereits mit Walsers Begleitbrief zum Manuskript wohl vom Spätsommer 1925. Darin fragt er an, ob Rychner den Text dafür geeignet hielte, in seiner "hochgeschätzten Zeitschrift irgendwo untergequetscht zu werden“ (B 651, S. 136). So betrachtet hat Rychner diese Anfrage bei der Publikation schlicht beim Wort genommen.

43 Vgl. Valéry, „Der Abend mit Herrn Teste“ (Anm. 38), S. 232.

44 Ernst Howald, „Die Psychologie Marcel Prousts“, NSR 19 (April 1926), S. 349-361; Paul Morand, „Ode an Marcel Proust“, ebd., S. 385 f.

45 Ernst Robert Curtius, „Saint-Evremond“, NSR 19 (März 1926), S. 288-30o. In den erhaltenen Briefen an Frieda Mermet lässt sich kein eindeutiger Beleg für die Behauptung finden, er habe ihr das Heft zukommen lassen; allerdings gibt es einen nicht ganz sicher auf den 16. Februar 1926 datierten Brief an sie, mit dem er ihr „eine Zeitschrift“ zuschickt, „worin 
Unvermittelt fährt Walsers Brief nach dieser rasanten Parade mit poetologischen Ausführungen zum Gedichteschreiben fort: „Ich fand die Frage Kerr's, ob zur Gedichtfabrikation ein Grad von Verblödung erwünscht sei, bemerkenswert" (B 685, S. 199). Die rätselhafte Passage belegt beiläufig, dass man weder Korrespondenzen noch Zeitschriften mittels des heuristischen Paradigmas einer Einzeltext- und -stellenphilologie und deren Vorstellungen von adressierbaren Bezügen gerecht wird. Denn vermutlich kann man tatsächlich lange und vergeblich in Alfred Kerrs Schriften nach der besagten Frage suchen; von und über Kerr ist im betreffenden Heft auch nichts abgedruckt. Jörg Schäfers und Robert Mächlers Kommentar zur Briefstelle lautet lakonisch: „Frage Kerr's: nicht ermittelt.“46 Aber zu ermitteln ist die Herkunft der „Frage Kerr's“ dennoch. Weit suchen muss man auch nicht. Man findet die Lösung des kleinen Rätsels nämlich in ebenjenem „sehr hübsche[n], interessante[n] Essay" über Saint-Evremond, den ausweichlich seines Briefes inzwischen die an und durch „Walser's Gesellschaft" gebildete „Glätterin“ Frieda Mermet liest:

Valéry sagte mir einmal, dass ihn seit langem ein Traktat beschäftige über das Thema: Séparation de la sottise et de la poésie. Und von Mallarmés Werk sagt er: ,On y voit se prononcer la tentative la plus audacieuse et la plus suivie qui ait jamais été faite pour surmonter ce que je nommerai l'intuition naive en littérature. Ein jüngerer Franzose, Louis Aragon, spricht ironisch von der ,forte faculté de crétiniser', welche den Charme der Poesie ausmache. Das Problem ein Kerrsches Problem - lautet also: ist Poesie ohne Verblödung möglich? Lyrik ohne Aberglauben? Die silberne Musik der Intelligenz in Valérys Versen gibt eine bezwingende Lösung.

Das dürfte eine Abschweifung gewesen sein. ${ }^{47}$

Um beinahe alle der vorher explizit und eindeutig adressierten Bezüge von Walsers Brief ließe sich mittels solcher Um- und Abwege ein weiter gefasstes Bezugsnetz spinnen, das die Referenzen zu einem Referenzraum erweitert. In der Passage über „Ablösung“ beziehungsweise „Einsetzung“ literarischkultureller „Weltkurfürsten“ hallt eine Bemerkung wider, die Rychner

auch ich mit einem Beitrag vertreten bin. Es sind zum Teil nette, lesenswerte Abschnitte in dem Blatt“" (B 68o, S. 186; vgl. die Erläuterungen zur Datierung).

46 Robert Walser, Briefe, hrsg. von Jörg Schäfer unter Mitarbeit von Robert Mächler, Frankfurt/Main: Suhrkamp 1979, S. 415.

47 Curtius, „Saint-Evremond“ (Anm. 45), S. 294f. Den Hinweis findet man jetzt auch im Kommentar der neuen Briefausgabe (zu B 685, S. 201) - allerdings ohne den wichtigen, da mit Walsers Poetik und konkret mit seiner im selben Heft abgedruckten Ruine korrespondierenden Kommentarsatz zitiert, mit dem Curtius zum anschließenden Absatz überleitet. 
seiner Rubrik zur Schweizer Literatur im Januar-Heft vorausgeschickt hat; ${ }^{48}$ das lakonische Urteil über Hiltbrunners Gedichte und insbesondere der anschließende Hinweis, dieses dem Betroffenen lieber zu verschweigen, spiegelt eine ihrerseits erkennbar skeptische Rezension von dessen 1925 erschienenem Gedichtband Winter und Wende wider, die man im letzten Heft des vorangehenden Zeitschriftenjahrgangs finden kann. ${ }^{49}$ Und wenn schließlich Walser in der Folge anfängt, aus der Kombination von ästhetischem Urteil und poetologischer Überlegung eine eigene, tentative Poetik der „Gedichtkörperbildung“ zu skizzieren (B 685, S. 199), dann erhellt daraus nur ein weiteres Indiz für die Produktivität solcher mobiler Kollektive, wie sie sich in Zeitschriftenheften kondensieren können - denn diese Poetik wiederum trägt mit ihrem Lob der beiläufigen Raffinesse deutliche Züge des von Curtius' Essay an Saint-Evremond ausgearbeiteten Literaturverständnisses. Eine solche Vernetzung ist strukturell unabschließbar, und so werden schon eine Woche später tatsächlich vier solcher „Gedicht-Körper“ (B 685, S. 119) an den „liebe[n], großmächtige[n] Herrn Dr. Rychner" gehen (B 687, S. 202), die auf drei Hefte des 20. Jahrgangs verteilt zwischen Juni und September 1927 in der Neuen Schweizer Rundschau gedruckt werden sollten. Sie nehmen die Rundschau"Revüe“ neu, unter formal und medial anderen Bedingungen noch einmal auf und setzen damit das kollaborative Unternehmen der Zeitschrift fort. ${ }^{50}$

Dass Walser mit poetologischer Scharfsicht und scharfsichtigem Witz zugleich auch beobachtet und kommentiert, was ein derartiges Unternehmen (an-)treibt, mag eine Ausnahme gegenüber dem ,Normalfall' solcher Zeitschriften-agency bilden. Als Anregung für eine Ästhetik des Kollaborativen, ${ }^{51}$

48 „In jedem Herbst befasst man sich mit soundsovielen Neuerscheinungen, und die vor drei Jahren an das strahlende Licht der Öffentlichkeit gesetzten Bücher muten die Neugier schon alt an. [...] Was blieb von den Werken, die auch einmal ihre große Stunde hatten und auch einmal im Mittelpunkt verschiedenen Interesses standen? Bücher altern schneller als Menschen, wenn sie nicht mit dem Ewigkeitszug gezeichnet sind“ (Max Rychner, „Zur Schweizer Literatur“, NSR 29 (Januar 1926), S. 92-104, hier S. 92).

49 „Das Buch hinterlässt den Eindruck: der Verfasser vermöge heut anscheinend spröden Formen ein sattes Leben einzuhauchen; nicht die Totalität, aber die Einzelheiten einer Landschaft fänden bei ihm oft überraschende Auslegung, das einzelne Wort selbst erhalte eine besondere Dichtigkeit; die von ihm umschriebenen menschlichen Vorgänge lassen hingegen da und dort die Frage offen: ob nicht manches in seiner Darstellung doch mehr hochgezwungen, hoch-gewünscht als geläutert sei? -“ (Siegfried Lang, „Gedichtbücher“, $W L 18$ (Dezember 1925), S. 1245-1256, hier S. 1249).

$50 \quad$ Robert Walser, „Couplet“, NSR 20 (Juni 1927), S. 621; ders., „Glosse“, NSR 20 (Juli 1927), S. 65o; ders., „Gedichte“, NSR 20 (September 1927), S. 885.

$5^{1}$ Den Begriff der ,Kollaboration', der der variablen Interdependenz funktional differenzierter Arbeitsprozesse möglicherweise eher gerecht wird als derjenige des ,Kollektivs', das stärker auf die gleichsam synekdochische Integration der an solchen Arbeitsprozessen 
unter deren konzeptuellem Dach sich künftige literaturwissenschaftliche Zeitschriftenerkundungen versammeln können, ist es ein Glücksfall. Auch jenseits der Neuen Schweizer Rundschau nämlich hat seine Aufmerksamkeit für die Spezifika solcher mobiler Kollektive nicht nachgelassen. In einem Brief an Hans Wilhelm Keller, Redakteur der anthroposophischen Individualität Anlass dafür ist einmal mehr die Zusendung eines Belegexemplars -, entwirft Walser eine regelrechte Poetik der Zeitschrift. Diese mache „einen guten Eindruck“ dann, wenn sie "gleichsam in ihrer äußeren Anordnung bildhaft, oder, man kann ebenso gut sagen, musikalisch“ sei. Ein „Zeitschriftenherausgeber“ solle auf „spielende, flötende Beiträge“ Wert legen, mit dem „Schwarz (Setzerschwärze) und Weiß (Kellnerinnenschürze oder unbedruckter Platz)" ihres (typo)graphischen Arrangements „Wirkungen der Interessantheit erzielen“. Für einen derartigen Herausgeber wählt der Brief die Metapher des „Dirigent[en]“ und bezieht noch die Lektüre in das ästhetische Kollaborationsprojekt ein: „Seite auf Seite müßte heute eine Zeitschrift sich auftun wie eine betörend duftende Blume, so, daß das Umblättern zum Ereignishaften würde; eine Bezauberung die andere ablöste. ${ }^{452}$ Eine solche Poetik in eine Analytik zurückzuübersetzen hieße, dem kollaborativen Schreiben von Zeitschriften und seinen Implikationen gerecht zu werden beginnen.

beteiligten Akteuren setzt, gälte es dabei zuallererst zu schärfen. Das allerdings muss einem eigenen Beitrag vorbehalten bleiben. In den nach Abschluss dieses Beitrags erschienenen Ausführungen von Carlos Spoerhase und Erika Thomalla, „Werke in Netzwerken“, in: Zeitschrift für deutsche Philologie 139 (2020), S. 145-163, wird der Kollaborationsbegriff, soweit ich sehe, analog zum Modell ,kollektiver Autorschaft' verwendet und dementsprechend ausschließlich auf die Verbindungen personaler Akteure bezogen.

$5^{2}$ Robert Walser an die Redaktion der Individualität/Hans Wilhelm Keller, o9.08.1926 (B 718, S. 241-244). Zum Brief vgl. Wolfram Groddeck, „Robert Walser an Hans Wilhelm Keller (1926)“, in: Briefe aus dem 20. Jahrhundert, hrsg. von Andreas Bernard und Ulrich Raulff, Frankfurt/Main: Suhrkamp 2005, S. 69-74. 